7. Patwardhan, R.V., Johnson, R.F., Hoyumpa, A. Jr. et al. Normal metabolism of morphine in cirrhosis. Gastroenterology 1981, 81: 1006-1011.

8. Gillman, M.A. \& Lichtigfeld, F.J. Analgesic nitrous oxide for alcohol withdrawal: critical assessment after 10 years use. Postgrad Med J 1990, 66: 543-546.

9. Cushman, P. Jr. Alcohol and opioids: possible interactions of clinical importance. Adv Alcohol Subst Abuse 1987, 6: 33-46.

\section{CT and MR imagings of single thyroid lobe (thyroid hemiagenesis) with Graves' disease}

Sir,

A case of aplasia of one thyroid lobe presenting with hyperthyroidism is described. To our knowledge this is the first reported study in which computed tomography (CT) and magnetic resonance imaging (MRI) have been used to diagnose hemiagenesis of the thyroid gland associated with Graves' disease.

A 28 year-old woman was referred to Fukuoka University Hospital in October 1990 for evaluation of a nodule in the left side of the neck associated with general fatigue, finger tremor and palpitation. She had been in good health until June 1989, when she began to notice increased nervousness, heat intolerance and palpitations. There was no history of excessive iodine intake or ingestion of medications, and no family history of thyroid disease.

On examination, the patient was anxious, pulse rate 98/min with warm and moist skin and a fine tremor of the hands. An enlarged and smooth nodule was palpated in the left lobe of the thyroid. The connecting isthmus was not palpable. No bruits or venous murmurs were heard and there was no evidence of Graves' ophthalmopathy. On clinical grounds she was thought to be hyperthyroid. Thyroid function tests showed: thyroxine (T4) $314.0 \mathrm{pmol} / \mathrm{l}$ (normal 51.2-153.6); triiodothyronine (T3) $5.9 \mathrm{nmol} / 1$ (normal 1.09-2.80); free T4 $87.52 \mathrm{pmol} / 1$ (normal 9.65-25.74); free T3 $5.9 \mathrm{nmol} / 1$ (normal 1.09-2.80); and thyrotrophin (TSH) less than $0.1 \mathrm{mU} / 1$ (normal $0.32-3.1$ ). Thyroid ${ }^{123}$ I-uptake was $62.8 \% / 24$ hours (normal 15-35) and scan revealed an enlarged left lobe with uniform and homogenous uptake; no right lobe was visualized (Figure 1). The image obtained with ${ }^{99 \mathrm{~m}}$ technetium was identical. Ultrasonography (US) of her neck, in addition to the palpable tissue in the left lobe, suggested an atrophic right lobe.

At that time we suspected Plummer's disease (toxic adenoma) with suppression of contralateral tissue. However, anti-microsomal antibody (MCHA) was slightly positive $(1: 400$ to $1: 800)$ and anti-thyroglobulin antibody (TGHA) was negative (1:100) (haemagglutination technique). TSH-binding inhibitor immunoglobulin (TBII; radioreceptor assay) ${ }^{1}$ and thyroid-stimulating antibody (TSAb; human thyroid adenylate cyclase stimulating activity $)^{2}$ were determined and both found positive; $42.7 \%$ and $569 \%$, respectively (normal $<15 \%$ and $<145 \%$, respectively). Computed tomography (Siemence DR2) and magnetic resonance imaging (Toshiba MRI200/TX, 1.5 tesla) were carried out for further evaluation and failed to reveal any right sided thyroid tissue. A diagnosis of thyroid hemiagenesis associated with Graves' disease was established and effective therapy with methimazole provided.

Thyroid hemiagenesis is a rare anatomical anomaly which may be found in the presence of any type of thyroid

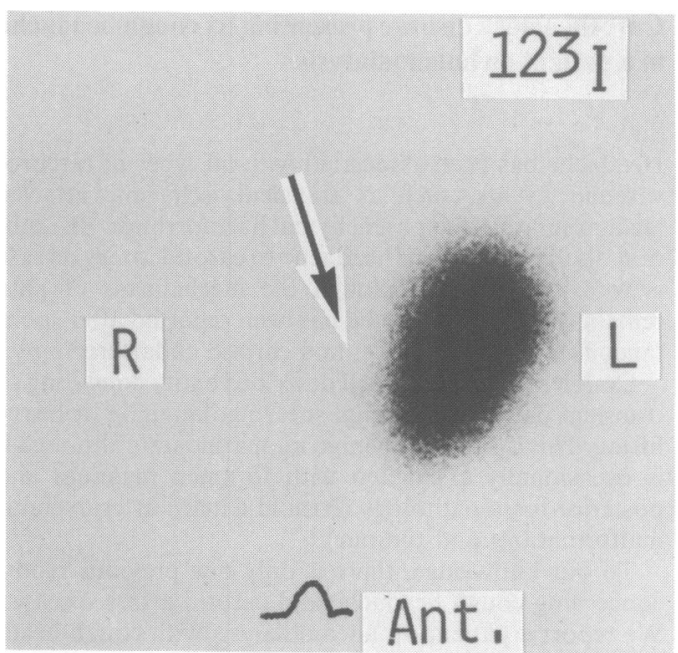

Figure $1{ }^{123}$ I thyroid scintigram.

disorder (adenomatous goitre, carcinoma, thyrotoxicosis and hypothyroidism). ${ }^{3-6}$ MRI and CT are useful in distinguishing betwen unilateral thyroid disease and true hemiagenesis. Moreover, the serum determinations of the anti-TSH receptor antibodies are also helpful in differentiation between Plummer's disease and hemiagenesis with superimposed Graves' disease.

Haruka Sasaki ${ }^{1,2}$

Tetsuhiro Futata ${ }^{2}$

Hirosi Ninomiya ${ }^{2}$

Yoshitaka Higashi ${ }^{3}$.

Makoto Okumura ${ }^{2}$

'Institute of Health Science,

Kyushu University,

Kasuga 816, Japan.

Departments of ${ }^{2}$ Internal Medicine and ${ }^{3}$ Radiology,

School of Medicine,

Fukuoka University,

Fukuoka 814-01, Japan.

\section{References}

1. Smith, B.R. \& Hall, R. Measurement of thyrotropin receptor antibodies. Methods Enzymol 1981, 74: 405-420.

2. Kasagi, K., Konishi, J., Iida, Y. et al. A new in-vitro assay for human thyroid stimulator using cultured thyroid cells: effects of sodium chloride on adenosine $3^{\prime} 5^{\prime}$-monophosphate increase. J Clin Endocrinol Metab 1982, 54: 108-114.

3. Melnick, J.C. \& Stemkowski, P.E. Thyroid hemiagenesis (Hockey stick sign): a review of the world literature and a report of four cases. J Clin Endocrinol Metab 1981, 52: 247-251.

4. Burman, K.D., Adler, R.A. \& Wartofsky, L. Hemiagenesis of the thyroid gland. Am J Med 1975, 58: 143-146.

5. De Remiges, P., D'Angelo, M., Bonaduce, S., Di Giandomenico, V. \& Sensi, S. Comparison of ultrasonic scanning and scintiscanning in the evaluation of thyroid hemiagenesis. J Clin Ultrasound 1985, 13: 561-563.

6. Mortimer, P.S., Tomlinson, I.W. \& Rosenthal, F.D. Hemiapladia of the thyroid with thyrotoxicosis. J Clin Endocrinol Metab 1981, 452: 152-155. 